(c) Elsevier/INRA

Article original

\title{
Effet de l'éthanol sur le temps de développement pré-imaginal de Drosophila melanogaster: étude de la variabilité géographique
}

\author{
H Merçot \\ Université $P \& M$ Curie, Laboratoire de génétique des populations, \\ UA 693 CNRS, Tour 42-4. 4, Place Jussieu, 75251 Paris Cedex 05, France
}

(Reçu le 25 mai 1990; accepté le 5 novembre 1990)

Summary - L'effet de l'éthanol sur le temps de développement pré-imaginal a été étudié dans des populations naturelles de Drosophila melanogaster originaires de France et d'Afrique. On observe un retard du développement pré-imaginal d'autant plus important que la concentration d'éthanol est élevée dans le milieu larvaire. Pour chaque population, le coefficient de régression du temps de développement sur la concentration d'éthanol $\left(b_{T D}\right)$ a été calculé. Ce coefficient est négativement corrélé à la latitude du lieu de piégeage des populations. Il en est de même avec la tolérance à l'éthanol de ces populations. Ainsi la meilleure adaptation des populations paléarctiques à un environnement alcoolisé se manifeste non seulement par une meilleure survie de l'œuf à l'adulte sur éthanol, mais également par une moindre sensibilité à l'effet retard de l'éthanol sur le développement pré-imaginal. Enfin, bien qu'une corrélation soit observée entre la valeur du coefficient $b_{T D}$ et la fréquence de l'allèle $A d h^{F}$, il n'est pas possible de dire s'il s'agit, pour ces 2 caractères, d'une relation de cause à effet ou d'un simple parallélisme de cline latitudinal.

Drosophila melanogaster / éthanol / temps de développement / variabilité géographique / Adh

\begin{abstract}
Summary - Ethanol effect on development time in Drosophila melanogaster: geographical variability. The effect of ethanol on pre-imaginal development time was studied in natural populations of Drosophila melanogaster from France and Africa. It was observed that the higher the concentration of ethanol in the larval medium, the more important was the delay in pre-imago development. For each population, the regression coefficient $\left(b_{T D}\right)$ of the development time on the ethanol concentration was computed. This coefficient was negatively correlated with the latitude of the trapping sites of the populations. Such was also the case with the ethanol tolerance of these populations. Thus, the better adaptation of the palearctic populations to an ethylic environment arises not only from a better egg-to-adult survival, but also from a lesser sensitivity to the delay-effect of ethanol on pre-imaginal development. Although a correlation between the $b_{T D}$ coefficient and the frequency of the $A d h^{F}$ allele was observed, it was not possible to determine whether these was a causal relationship between the 2 traits or whether this was due to a latitude factor.
\end{abstract}

Drosophila melanogaster / ethanol / development time / geographical variability / Adh 


\section{INTRODUCTION}

L'espèce Drosophila melanogaster est, parmi les espèces du genre Drosophila, l'une des mieux adaptées à la vie sur des substrats à fermentation alcoolique (Atkinson et Shorrocks, 1977; Monclus et Prevosti, 1978-79; David et Van Herrewege, 1983). L'effet principal de l'éthanol est de causer une mortalité des individus qui s'accroît avec la concentration du produit (Merçot et Charles-Palabost, 1987, pour références). Tant pour la tolérance des adultes que pour la survie de l'œuf à l'adulte, on observe chez cette espèce une grande variabilité géographique en liaison avec la latitude du lieu de capture des populations, la tolérance augmentant avec la latitude (David et al, 1986).

L'action délétère de l'éthanol se traduit également par un retard du développement pré-imaginal (Oakeshott, 1976; Parsons et al, 1979; Dorado et Barbancho, 1984; Higuet, 1984; Merçot et Charles-palabost, 1987; Van Herrewege et David, 1987). L'objet du présent travail a été de déterminer s'il existait, comme pour la mortalité sur éthanol, une variabilité géographique de la réponse à l'effet retard de l'éthanol sur le développement pré-imaginal.

\section{MATÉRIEL ET MÉTHODES}

Douze populations naturelles de Drosophila melanogaster originaires de France et d'Afrique ont été étudiées (voir tableau I).

Les lieux de capture se répartissent dans une zone allant de $33,5^{\circ}$ de latitude Sud (le Cap) à $49,0^{\circ}$ de latitude Nord (Venteuil) et de $17,3^{\circ}$ de longitude Ouest (Dakar) à $28,0^{\circ}$ de longitude Est (Johannesbourg). Moins de 3 mois se sont écoulés entre le piégeage des populations et le début des tests, excepté pour la population Brazzaville, capturée 1 an auparavant.

C'est au cours d'une expérience de survie de l'œuf à l'adulte sur éthanol (avec détermination de la concentration létale 50 d'éthanol $\left(\mathrm{CL}_{50}\right)$ ) (David et al, 1986) que l'étude du temps de développement a été réalisée pour différentes concentrations d'éthanol (voir tableau I). Le milieu de développement pré-imaginal est un milieu axénique (levure de bière sèche $70 \mathrm{~g}$, sucrose $70 \mathrm{~g}$, agar $20 \mathrm{~g}$, nipagine $6 \mathrm{~g}$, eau 11) additionné d'éthanol à la concentration souhaitée. L'éthanol est vigoureusement mélangé au milieu lorsque celui est à une température de $50^{\circ} \mathrm{C}$. Le tout est coulé dans des tubes (à raison de $9 \mathrm{ml}$ par tube) stockés à $6^{\circ} \mathrm{C}$ et remis à température ambiante $18 \mathrm{~h}$ plus tard en vue de l'expérience. Celle-ci a consisté à étaler, par tube, 50 œufs âgés de 0-3 h. Pour chaque population et chaque concentration testée, 5 tubes répliques ont été constitués. Après étalement, les tubes, bouchés par du coton cardé, ont été placés dans une étuve à $25^{\circ} \mathrm{C}$ et $70 \%$ d'humidité avec une photopériode de $12 \mathrm{~h}$. L'éthanol étant un produit volatile, cette technique n'évite pas l'évaporation progressive de l'éthanol au cours du temps. À l'émergence des imagos, leur dénombrement a été réalisé toutes les $24 \mathrm{~h}$. Pour chaque tube, la moyenne géométrique du temps de développement a été calculée sur l'ensemble des imagos, sans distinction des sexes. Pour une concentration donnée, le temps de développement $(T D)$ a été estimé à partir de la moyenne des 5 moyennes géométriques, sous réserve que le pourcentage d'émergence dans chacun des 5 tubes répliques ait été $\geq 10$. Le coefficient de régression $\left(b_{T D / E}\right)$ du temps de 
Table I. Temps de développement en jours ( $T D$; es = erreur standard) et pourcentage d'imagos émergés (ie\%) aux différentes concentrations d'éthanol pour les 12 populations testées.

\begin{tabular}{|c|c|c|c|c|c|c|c|c|c|c|}
\hline \multirow{2}{*}{$\begin{array}{l}\text { Localité } \\
\text { (Pays) } \\
\text { Latitude }\end{array}$} & & \multicolumn{9}{|c|}{ Concentrations en éthanol du milieu d'élevage (\%) } \\
\hline & & 0 & 4 & 6 & 8 & 10 & 12 & 14 & 15 & 18 \\
\hline Venteuil & $T D$ & 10,33 & 10,62 & & 11,29 & & 12,34 & & 13,54 & 14,71 \\
\hline (France) & es & 0,09 & 0,12 & & 0,14 & & 0,05 & & 0,15 & 0,18 \\
\hline $49,0 \mathrm{~N}$ & $\mathrm{ie} \%$ & 90,00 & 86,80 & & 87,20 & & 84,80 & & 69,60 & 33,20 \\
\hline Chateaubriand & $T D$ & 10,36 & 10,95 & & 11,64 & & 12,80 & & 13,39 & 14,29 \\
\hline (France) & es & 0,07 & 0,08 & & 0,08 & & 0,15 & & 0,22 & 0,26 \\
\hline $47,4 \mathrm{~N}$ & $\mathrm{ie} \%$ & 82,00 & 86,80 & & 73,20 & & 70,00 & & 44,80 & 36,80 \\
\hline Ménétréol & $T D$ & 10,27 & 10,82 & & 11,32 & & 12,41 & & 13,34 & 13,94 \\
\hline (France) & es & 0,04 & 0,09 & & 0,08 & & 0,13 & & 0,05 & 0,05 \\
\hline $47,2 \mathrm{~N}$ & & & 91,60 & & 92,40 & & 79,60 & & 64,40 & 34,80 \\
\hline Bonnac-la-Côte & $T D$ & 10,19 & 10,72 & & 11,49 & & 12,34 & & 13,79 & 15,17 \\
\hline (France) & es & 0,04 & 0,06 & & 0,07 & & 0,07 & & 0,25 & 0,26 \\
\hline $45,5 \mathrm{~N}$ & $\mathrm{ie} \%$ & 87,00 & 85,60 & & 82,40 & & 74,80 & & 49,20 & 16,00 \\
\hline Banyuls & $T D$ & 10,24 & 10,77 & & 11,35 & & 12,58 & & 13,37 & 14,71 \\
\hline (France) & es & 0,03 & 0,06 & & 0,05 & & 0,03 & & 0,12 & 0,10 \\
\hline $42,3 \mathrm{~N}$ & ie\% & 88,33 & 88,00 & & 84,00 & & 72,80 & & 57,76 & 44,00 \\
\hline Bou-Argoub & $T D$ & 10,19 & 10,83 & & 11,45 & & 12,70 & & 13,09 & 14,38 \\
\hline (Tunisie) & es & 0,02 & 0,04 & & 0,09 & & 0,15 & & 0,10 & 0,10 \\
\hline $36,2 \mathrm{~N}$ & $i e \%$ & 95,33 & 94,80 & & 93,60 & & 84,50 & & 65,20 & 37,00 \\
\hline Dakar & $T D$ & 10,33 & 10,80 & 11,43 & 11,57 & 12,43 & 13,22 & 14,13 & & \\
\hline (Sénégal) & es & 0,05 & 0,06 & 0,18 & 0,11 & 0,16 & 0,25 & 0,20 & & \\
\hline $14,5 \mathrm{~N}$ & $\mathrm{ie} \%$ & 78,00 & 80,00 & 74,80 & 75,20 & 61,20 & 41,20 & 25,60 & & \\
\hline Cotonou & $T D$ & 10,05 & 10,70 & 11,55 & 12,29 & 13,15 & & & & \\
\hline (Bénin) & es & 0,03 & 0,07 & 0,19 & 0,20 & 0,36 & & & & \\
\hline $6,2 \mathrm{~N}$ & ie\% & 71,33 & 71,20 & 63,20 & 28,80 & 10,00 & & & & \\
\hline Lamto & $T D$ & 10,53 & 11,04 & 11,54 & 12,02 & 12,63 & & & & \\
\hline (Cte d'Iv) & es & 0,05 & 0,14 & 0,11 & 0,04 & 0,09 & & & & \\
\hline $6,1 \mathrm{~N}$ & ie\% & 73,33 & 66,00 & 69,60 & 60,00 & 28,40 & & & & \\
\hline Brazzaville & $T D$ & 10,68 & 11,81 & 12,53 & 13,31 & 14,29 & & & & \\
\hline (Congo) & es & 0,08 & 0,07 & $0,1.0$ & 0,12 & 0,23 & & & & \\
\hline $4,2 \mathrm{~S}$ & ie\% & 78,00 & 67,20 & 72,40 & 61,60 & 14,80 & & & & \\
\hline Johannesbourg & $T D$ & 10,26 & 10,73 & & 11,81 & & 14,04 & & & \\
\hline (Afr du Sud) & es & 0,02 & 0,05 & & $\begin{array}{r}0,09 \\
700\end{array}$ & & 0,20 & & & \\
\hline $26,2 \mathrm{~S}$ & $\mathrm{ie} \%$ & 80,33 & 79,60 & & 70,80 & & 21,20 & & & \\
\hline Le Cap & $T D$ & 10,28 & 10,78 & & 11,36 & & 12,89 & & 13,26 & \\
\hline (Afr du Sud) & es & 0,03 & 0,03 & & 0,04 & & 0,14 & & 0,09 & \\
\hline $33,5 \mathrm{~S}$ & $\mathrm{ie} \%$ & 74,67 & 73,60 & & 68,80 & & 38,40 & & 17,60 & \\
\hline
\end{tabular}


développement sur la concentration en éthanol a été calculé après transformation logarithmique de $T D$ dans le but de normaliser sa distribution et de linéariser la droite de régression.

\section{RÉSULTATS}

Le tableau I donne, pour les 12 populations, les valeurs du temps de développement $(T D)$ et le pourcentage d'adultes éclos aux différentes concentrations testées.

Il est essentiel de considérer ces résultats en fonction de la technique utilisée, à savoir l'addition d'éthanol au début de l'expérience. En effet celle-ci n'évite pas l'évaporation progressive de l'éthanol au cours du temps. Van Herrewege et David (1987) ont observé qu'un milieu préparé à $10 \%$ d'éthanol voyait cette concentration réduite à $2 \%$ après $10 \mathrm{j}$ à la température de $25^{\circ} \mathrm{C}$. Ce sont donc essentiellement le stade embryonnaire et les 2 premiers stades larvaires qui sont affectés par la présence d'éthanol. Cette technique qui est la plus couramment utilisée, tant pour étudier la survie de l'œuf à l'adulte sur éthanol (Merçot et Charles-Palabost, pour référence) que les effets de cet alcool sur le temps de développement pré-imaginal (Oakeshott, 1976; Parsons et al, 1979; Dorado et Barbancho, 1984; Higuet, 1984; Merçot et Charles-Palabost, 1987), diffère donc essentiellement de celle développée par Van Herrewege et David (1987) et qui consiste à maintenir constante la concentration d'éthanol au cours du temps. Par cette dernière technique, tous les stades pré-imaginaux sont également confrontés à l'éthanol. Ceci a pour conséquence de provoquer une forte mortalité dès les plus faibles concentrations d'éthanol, concentrations jugées sans effets si l'on utilise la première méthode.

Pour en revenir à nos résultats, on observe, en l'absence d'éthanol, une différence significative du temps de développement entre les 12 populations (test de KruskallWallis: $H=39,57,11 \mathrm{ddl} ; P<0,001)$. On observe une relation négative entre temps de développement et latitude du lieu de piégeage, mais celle-ci n'est pas statistiquement significative $(r=-0,368 ; 10 \mathrm{ddl}$; ns), ce qui corrobore les observations faites par David et al (1976) sur ce type de populations.

La présence d'éthanol accroît le temps de développement et ceci dès la concentration $4 \%$ (tableau I) (test de Wilcoxon pour séries appariées: $T=0 ; 12 \mathrm{ddl}$; $P<0,01$ ), alors que le pourcentage d'émergence n'est pas globalement diminué à cette concentration par rapport au milieu sans alcool $(T=18 ; 12 \mathrm{ddl}$; ns $)$. Cependant pour de plus faibles concentrations (1 et $2 \%$ ) Parsons et al (1979) ont observé une réduction du temps de développement, résultat dû peut-être aux effets trophiques de l'éthanol à faible concentration (Van Herrewege and David, 1980; Geer et al, 1986).

Les coefficients de régression du temps de développement sur la concentration d'éthanol $\left(b_{T D / E}\right)$ permettent de quantifier l'accroissement du retard occasionné par la présence d'alcool (tableau II), et ceci indépendamment de la valeur du temps de développement à la concentration 0 . Selon ce critère, la sensibilité d'une population à l'éthanol est d'autant plus grande que la valeur du coefficient de régression $b_{T D / E}$ est élevée. Le tableau II donne également la valeur des doses létales 50 d'éthanol pour la survie de l'œuf à l'adulte ainsi que la fréquence de l'allèle Fast $\left(A d h^{F}\right)$ du gène de l'alcool déshydrogénase, puisqu'il s'agit du gène dont 
l'enzyme $(\mathrm{ADH})$ assure la détoxification de l'éthanol dans l'organisme de la mouche. À partir de ces données, nous avons calculé les coefficients de corrélation entre le coefficient de régression $b_{T D / E}$ et respectivement la latitude, la $\mathrm{CL}_{50}$ d'éthanol et la fréquence de l'allèle $A d h^{F}$. La valeur du coefficient $b_{T D / E}$ est négativement corrélée avec la latitude $(r=-0,620,10 \mathrm{ddl} ; P<0,05)$. Le retard du développement préimaginal provoqué par l'éthanol est d'autant plus grand que l'on considère des populations plus proches de l'équateur, avec cependant une exception concernant la population de Lamto (tableau II). Le coefficient $b_{T D / E}$ est également corrélé avec la $\mathrm{CL}_{50}$ d'éthanol $(r=-0,692,10 \mathrm{ddl} ; P<0,05)$ et la fréquence de l'allèle $A d h^{F}$ $(r=-0,667,10 \mathrm{ddl} ; P<0,05)$. Ces 2 derniers résultats ne sont pas surprenants puisque la tolérance à l'éthanol et la fréquence de l'allèle $A d h^{F}$ sont connus pour varier selon un cline latitudinal (David et al, 1986).

Table II. Survie de l'œuf à l'adulte $\left(\mathrm{CL}_{50}\right.$ éthanol); coefficient de régression $\left(b_{T D / E}\right)$ du temps de développement pré-imaginal sur la concentration en éthanol et fréquence de l'allèle $A d h^{F}$ dans les 12 populations naturelles de Drosophila melanogaster.

\begin{tabular}{lrrrr}
\hline Localité & Latitude & $C L_{50} \pm \sigma$ & $b_{T D / E} \cdot 10^{3} \pm \sigma$ & $A_{d h^{F}}$ \\
\hline Venteuil & $49,0 \mathrm{~N}$ & $17,0 \pm 0,3$ & $7,76 \pm 0,50$ & 0,935 \\
Châteaubriand & $47,4 \mathrm{~N}$ & $16,6 \pm 0,4$ & $7,86 \pm 0,31$ & 0,991 \\
Ménétréol & $47,2 \mathrm{~N}$ & $16,7 \pm 0,3$ & $7,65 \pm 0,25$ & 0,949 \\
Bonnac-la-Côte & $45,5 \mathrm{~N}$ & $15,0 \pm 0,2$ & $8,43 \pm 0,44$ & 0,938 \\
Banyuls & $42,3 \mathrm{~N}$ & $18,0 \pm 0,7$ & $8,58 \pm 0,26$ & 0,918 \\
Bou-Argoub & $36,2 \mathrm{~N}$ & $16,8 \pm 0,3$ & $8,08 \pm 0,25$ & 0,873 \\
Dakar & $14,5 \mathrm{~N}$ & $12,2 \pm 0,2$ & $9,79 \pm 0,53$ & 0,360 \\
Cotonou & $6,2 \mathrm{~N}$ & $7,6 \pm 0,1$ & $11,85 \pm 0,89$ & 0,016 \\
Lamto & $6,1 \mathrm{~N}$ & $9,6 \pm 0,3$ & $7,94 \pm 0,47$ & 0,090 \\
Brazzaville & $4,2 \mathrm{~S}$ & $8,5 \pm 0,2$ & $12,54 \pm 0,51$ & 0,064 \\
Johannesburg & $26,2 \mathrm{~S}$ & $10,2 \pm 0,2$ & $11,23 \pm 0,81$ & 0,337 \\
Le Cap & $33,5 \mathrm{~S}$ & $11,8 \pm 0,3$ & $7,86 \pm 0,37$ & 0,519 \\
\hline
\end{tabular}

\section{DISCUSSION}

La durée du développement est un caractère dépendant de l'expression et de l'interaction d'un grand nombre d'unités génétiques, unités sans doute impliquées dans l'adaptation à différents types d'environnement (Church et Robertson, 1966; Cluster et al, 1987). C'est un caractère de première importance pour la vie d'une mouche car il influe directement sur la compétition larvaire. En effet, les larves à développement plus lent, subissant des conditions alimentaires plus dures, peuvent voir, suivant la nature du milieu, leur retard s'accentuer et/ou leur viabilité diminuer par rapport aux larves à développement plus rapide (Anxolabéhère, 1978).

Comme d'autres toxiques (David, 1963; Le Menn et al, 1983; Botella et al, 1983, 1984) l'éthanol provoque un retard du développement pré-imaginal, retard s'observant même à des concentrations non létales. Cet effet retard est négativement 
corrélé à la latitude, et à la $\mathrm{CL}_{50}$ d'éthanol. La meilleure adaptation des populations françaises de Drosophila melanogaster à l'éthanol par rapport aux populations africaines s'illustre donc par une plus faible mortalité (valeurs de $\mathrm{CL}_{50}$ d'éthanol plus élevées; tableau II), et un développement pré-imaginal moins retardé. Ces résultats montrent que la sélection naturelle a joué en faveur de facteurs atténuant l'effet retard tout comme elle a agi en faveur d'une augmentation de la tolérance à l'éthanol. On peut dès lors émettre 2 hypothèses:

- la sélection a agi sur un seul système génétique favorisant conjointement une meilleure tolérance à l'éthanol et une moindre sensibilité à son effet retard;

- les 2 caractères sont, en tout ou partie, génétiquement indépendants, une sélection simultanée sur ces 2 caractères ayant permis une meilleure adaptation à la présence d'éthanol dans l'environnement.

Il n'est pas possible de trancher entre ces 2 hypothèses. Mais si l'on privilégie la première, peut-elle s'appliquer au polymorphisme de l'Alcool déshydrogénase? Cluster et al (1987) ont observé, après sélection pour un temps de développement plus court, une augmentation de l'activité de 3 enzymes (alcool déshydrogénase, $\alpha$-Glycérophosphate déshydrogénase, enzyme malique) sur 4 étudiées. Ici, l'étude a porté sur l'effet retard d'un produit exogène, l'éthanol, sur le développement préimaginal. Elle aboutit cependant à un résultat semblable : ce sont les populations où la fréquence de l'allèle $A d h^{F}$ - donc de l'alloenzyme la plus active - (Day et al, 1974; Lewis et Gibson, 1978; McDonald et al, 1980) est la plus élevée qui subissent le moins de retard. Cependant cette corrélation est moins forte que celle existant entre la $\mathrm{CL}_{50}$ et la fréquence de l'allèle $A d h^{F}(r=0,964,10 \mathrm{ddl} ; P<0,01)$. Comme dans le cas de la tolérance à l'éthanol (David et al, 1986), il n'est pas possible de trancher entre une relation de cause à effet et un simple parallélisme de cline latitudinal.

\section{REMERCIEMENTS}

C'est grâce aux Drs D Anxolabéhère, P Capy, J David, D Lachaise, C Montchamp-Moreau et $\mathrm{S}$ Ronsseray que j'ai pu disposer des populations de $D$ melanogaster analysées dans ce travail. Je remercie le Dr J David et l'expert anonyme qui m'ont permis, par leurs commentaires, d'améliorer ce manuscrit.

\section{RÉFÉRENCES}

Anxolabéhère D (1978) Analyse expérimentale et théorique du rôle des valeurs sélectives variables dans le maintien du polymorphisme au locus sepia chez Drosophila melanogaster. Thèse de Doctorat d'Etat, Univ Paris VII, $173 \mathrm{p}$

Atkinson W, Shorrocks B (1977) Breeding site specificity in the domestic species of Drosophila. Ecologia 29, 223-232

Botella LM, Moya A, Mensua JL (1983) Effect of urea on viability and mean development time of Drosophila melanogaster larvae. Dros Inf Serv 59, 23-24

Botella LM, Moya A, Mensua JL (1984) Effect of butyrate on the development of D melanogaster. Dros Inf Serv 60, 67-68

Church RB, Robertson FW (1966) Biochemical analysis of genetic differences in the growth of Drosophila. Genet Res 7, 383-407 
Cluster PD, Marinkovic D, Allard RW, Ayala FJ (1987) Correlations between development rates, enzyme activities, ribosomal DNA spacer-length phenotypes, and adaptation in Drosophila melanogaster. Proc Natl Acad Sci USA 84, 614-610 David J (1963) Les effets physiologiques de l'intoxication par le bleu de méthylène sur la Drosophile. Bull Biol Fr Belg 97, 515-530

David JR, Van Herrewege J (1983) Adaptation to alcoholic fermentation in Drosophila species: relationship between alcohol tolerance and larval habitat. Comp Biochem Physiol 74A, 283-288

David J, Bocquet C, Pla E (1976) New results on the genetic characteristics of the Far East race of Drosophila melanogaster. Genet Res 28, 253-260

David JR, Merçot H, Capy P, McEvey SF, Van Herrewege J (1986) Alcohol tolerance and $A d h$ gene frequencies in European and African populations of Drosophila melanogaster. Génét Sél Evol 18, 405-416

Day TH, Hillier PC, Clarke B (1974) The relative quantities and catalytic activities of enzymes produced by alleles at the alcohol dehydrogenase locus in Drosophila melanogaster. Biochem Genet 11, 155-165

Dorado G, Barbancho M (1984) Differential response in Drosophila melanogaster to the environmental ethanol modification of fitness components at the $A d h$ locus. Heredity 53, 309-320

Geer BW, McKechnie SW, Langevin ML (1986) The effect of dietary ethanol on the composition of lipids of Drosophila melanogaster larvae. Biochem Genet 24, 51-69

Higuet D (1984) Réponse d'une population naturelle de Drosophila melanogaster $\grave{a}$ une sélection disruptive sur le poids, selon le mode de croisement. Thèse de $3^{\mathrm{e}}$ Cycle, Univ Paris VII, $118 \mathrm{p}$

Le Menn A, Silber J, Goux J-M (1983) Etude de l'effet de deux inhibiteurs de la synthèse des nucléotides, l'aminoptérine et la fluorodésoxyuridine, sur des souches sauvages et mutantes vestigial chez Drosophila melanogaster. Biol Cell 49, 213-218 Lewis N, Gibson J (1978) Variation in amount of enzyme protein in natural population. Biochem Genet 16, 159-170

McDonald JF, Anderson SM, Santos M (1980) Biochemical differences between products of the Adh locus in Drosophila. Genetics 95, 1013-1022

Merçot H, Charles-Palabost L (1987) Effet de l'éthanol sur le développement préimaginal de Drosophila melanogaster: relation avec les locus de l'Adh et de l' $\alpha$ Gpdh. Génét Sél Evol 19, 413-426

Monclus M, Prevosti A (1978-79) Cellar habitat and Drosophila populations. Génét Ibér 30-31, 189-201

Oakeshott JG (1976) Selection at the alcohol dehydrogenase locus in Drosophila melanogaster imposed by environmental ethanol. Genet Res 26, 265-274

Parsons PA, Stanley SM, Spence GE (1979) Environmental ethanol at low concentrations: longevity and development in the sibling species Drosophila melanogaster and $D$ simulans. Aust $J$ Zool 27, 747-754

Van Herrewege J, David JR (1980) Alcohol tolerance and alcohol utilization in Drosophila: partial independence of two adaptative traits. Heredity 44, 229-235 Van Herrewege J, David JR (1987) Effects of ethanol on the development of Drosophila melanogaster: comparison of two geographic populations. $J$ Insect Physiol 33, 893-898 